\title{
Investigation of REST-class Hypersonic Inlet Designs
}

\author{
Rowan J. Gollan* and Paul G. Ferlemann ${ }^{\dagger}$ \\ NASA Langley Research Center, Hampton, Virginia
}

\begin{abstract}
Rectangular-to-elliptical shape-transition (REST) inlets are of interest for use on scramjet engines because they are efficient and integrate well with the forebody of a planar vehicle. The classic design technique by Smart for these inlets produces an efficient inlet but the complex three-dimensional viscous effects are only approximately included. Certain undesirable viscous features often occur in these inlets. In the present work, a design toolset has been developed which allows for rapid design of REST-class inlet geometries and the subsequent Navier-Stokes analysis of the inlet performance. This gives the designer feedback on the complex viscous effects at each design iteration. This new tool is applied to design an inlet for on-design operation at Mach 8. The tool allows for rapid investigation of design features that was previously not possible. The outcome is that the inlet shape can be modified to affect aspects of the flow field in a positive way. In one particular example, the boundary layer build-up on the bodyside of the inlet was reduced by $20 \%$ of the thickness associated with the classically designed inlet shape.
\end{abstract}

\section{Nomenclature}

$A \quad$ wetted surface area of the inlet, $\mathrm{m}^{2}$

$A_{\delta} / A_{c}$ boundary layer area ratio (proportion of boundary layer area, $A_{\delta}$, at a given cross-section, $A_{c}$ )

$D \quad$ drag force, $\mathrm{N}$

$F \quad$ stream thrust, N

$M \quad$ Mach number

$\dot{m} \quad$ mass flow rate, $\mathrm{kg} / \mathrm{s}$

$p \quad$ static pressure, $\mathrm{Pa}$

$q \quad$ dynamic pressure, $\mathrm{Pa}$

$T \quad$ static temperature, $\mathrm{K}$

$\delta \quad$ boundary layer thickness

$\delta^{*} \quad$ displacement thickness

$\theta \quad$ momentum thickness

$\theta_{b} \quad$ forebody angle, degrees

$\tau \quad$ shear stress, $\mathrm{Pa}$

Subscripts

0 free stream, flight conditions

1 inlet entrance conditions

${ }^{*}$ Research Scholar, National Institute of Aerospace

${ }^{\dagger}$ ATK, Space Systems Group 


\section{Introduction}

Rectangular-to-elliptical shape transition (REST) inlets are a class of three-dimensional hypersonic inlet having the desirable feature of tight integration with a planar vehicle body. Integration with a planar forebody is desirable since the flow angle approaching the inlet is not affected by vehicle angle-of-attack. These quasi-streamline-traced inlets are efficient, and, by utilizing a shape transition, are able to connect to various combustor shapes. An example of a modular arrangement of three REST inlets is shown in Figure 1. The classic design approach by Smart $^{1}$ for these inlets cannot fully account for the complex three-dimensional boundary layer phenomena - these viscous effects alter the inlet flow such that the high efficiency of the inviscid core flow is not fully realized. It is believed that using high-fidelity analysis in this case, Navier-Stokes computational fluid dynamics (CFD) calculations - early in the design process allows the inlet designer to better account for the viscous effects and, subsequently, to use this knowledge to improve the inlet performance.

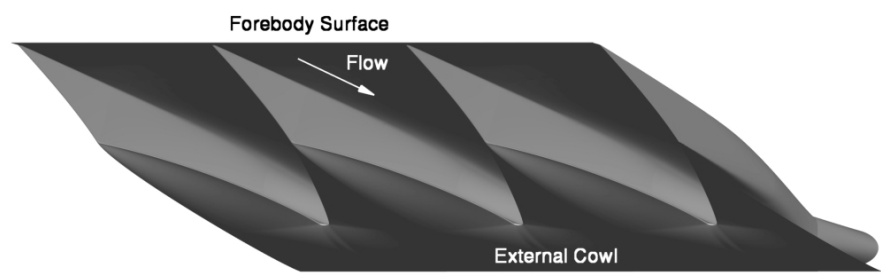

(a) view from bottom

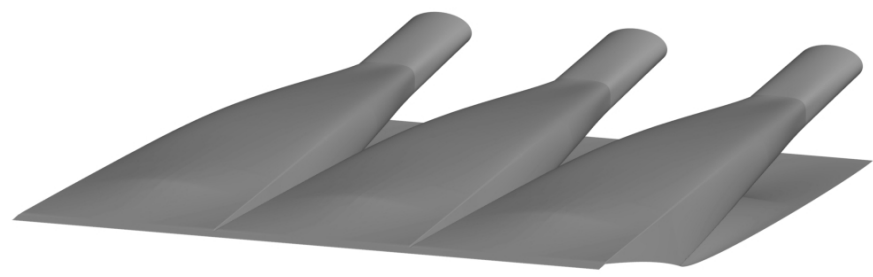

(b) view from top

Figure 1. Three REST inlets shown in a modular arrangement, from two views. Source: Ferlemann and Gollan ${ }^{2}$

The classic REST inlet design method was first presented by Smart ${ }^{1}$ in 1999. Smart demonstrated a method that utilized lofting of streamline-traced inlets to produce a shape-transitioning inlet. The streamline-tracing method is based on inviscid flow fields, and then the inlet surface is corrected (that is, enlarged) to account for boundary layer growth. One limitation of this design method is that it produces inlet geometries for only a restricted portion of the design space for this class of inlet. The procedure may be iterated by varying some input parameters, but the resulting surfaces are confined to a small portion of the design space as dictated by the nature of the method. For example, one problem that arises from this approach is that there is an unfavorable boundary layer roll-up that develops in the inlet. This undesirable flow feature can limit the back pressure capability and affect robust operation of the engine. To address this particular problem and other aspects of the inlet flow field, a means to alter the inlet surface that is divorced from the classic design procedure used to generate the surface is required. In this way, a larger portion of the design space is made available.

Recent work by Ferlemann and Gollan ${ }^{2}$ has focused on developing tools that allow the inlet geometry to be manipulated in a parametric way that is meaningful from an inlet design perspective. Additionally, this new toolset provides a means to move rapidly from altering surface geometry to the generation of a volume grid ready for a Navier-Stokes CFD calculation.

This paper investigates the application of this advanced toolset for the design and analysis of REST-class 
hypersonic inlets. A special feature of this toolset is the use of high-fidelity analysis upfront, at the early stages of design. This has given new insights and a better appreciation for the complex REST inlet flow field. As the main focus of the paper, the toolset is exercised to design a REST inlet for use in a $M_{0}=8$ scramjet cruise application. The goal was to positively influence an inlet metric using the design toolset and compare to an inlet design based on the classic approach. In particular, the choice was made to reduce the thickness of the boundary layer on the inlet bodyside centerline before the isolator because an increased thickness can limit the ability of the inlet to withstand the combustor-induced pressure rise.

\section{Design tool}

Part of the difficulty of including high-fidelity analysis in the design process is the tedious and timeconsuming task of preparing grids for the CFD calculation. A large amount of effort has been, and continues to be, focused on automating as much of the grid generation for these REST-class inlet designs as possible. This allows the designer to spend time on design decisions rather than on the generation of grids. The sequence of steps in one design iteration includes: (1) specification of an inlet geometry, in parametric form; (2) preparation of surfaces from the parametrized geometry; (3) generation of a structured volume grid based on the surfaces; and (4) analysis of the inlet performance using Navier-Stokes CFD calculations.

\section{Classic REST design method}

In the sequence of steps above, the first step involves specifying an inlet geometry appropriate for the target design parameters. The classic REST design method is used to start the iterative design process. The classic design method is strongly tied to tracing through a specific compression field. This compression field is selected based on an inlet Mach number at on-design flight conditions. Thus, the resulting quasi-streamlinetraced inlet has characteristics which are appropriate for the design Mach number condition, such as turning angles and total contraction ratio. This is a very convenient starting point for specifying an inlet geometry compared to the designer trying to develop a set of angles and amount of geometric contraction. In other words, the classic REST design process captures aspects of the physics that are not included in the purely geometric parametric representation of the inlet surface.

The classic REST design method has been documented extensively by Smart. ${ }^{1}$ Here a brief overview of the procedure is given. The design method begins with the specification of a compression field. The compression field should result in a target throat pressure based upon the on-design inflow conditions. It is also desirable to choose a compression field that is as efficient as possible, as the final design shape is trying to recover an inviscid core flow which is close to this initial compression field. Having chosen the compression field, various inlets are formed by tracing streamlines through the flow field. For example, one inlet is traced in the flow direction (downstream) from a specified rectangular-like capture shape which can be attached to a planar vehicle forebody. A second inlet is traced in the reverse direction (upstream) from an assumed throat location, using a specified throat shape; in this case, an ellipse. These inlet shapes are then blended using a lofting technique such that there is a shape transition from a rectangular-like capture shape down the length of the inlet to become an elliptical throat. In practice, additional streamline-traced inlets may be blended to control the inlet shape at various intermediate locations.

At this point, the design method has only considered the inviscid flow physics. The surface is then corrected for viscous effects. A boundary layer displacement thickness is computed using an approximate method. This thickness is added to the surface to enlarge it, and thus correct for the viscous effects of boundary layer growth. Finally, the surface is smoothed since the viscous correction gives rise to some uneveness.

\section{Parametric geometry representation of the inlet surface}

The inlet surface resulting from the classic design method is represented as a 'cloud of points'. To facilitate the remainder of the design and analysis process, it is desirable to have a parametric computer-aided design (CAD) geometry of the inlet. The Adaptive Modeling Language ${ }^{3}$ (AML) is used to construct a parametric CAD geometry that represents the cloud of points defining the inlet. The parametric representation uses a series of curves to capture essential features of the inlet shape. An example of the parametric curves defining the inlet is shown in Figure 2. The light gray curves in Figure 2 are auxiliary curves that are built by the system: they are not directly specified by the user. These curves form the basis of lofted surface patches, 
and these surface patches are stitched together to give a final surface shape. As part of the parametrization, some 'real-world' design parameters are introduced, such as a leading edge radius. An example surface is also shown in Figure 2.

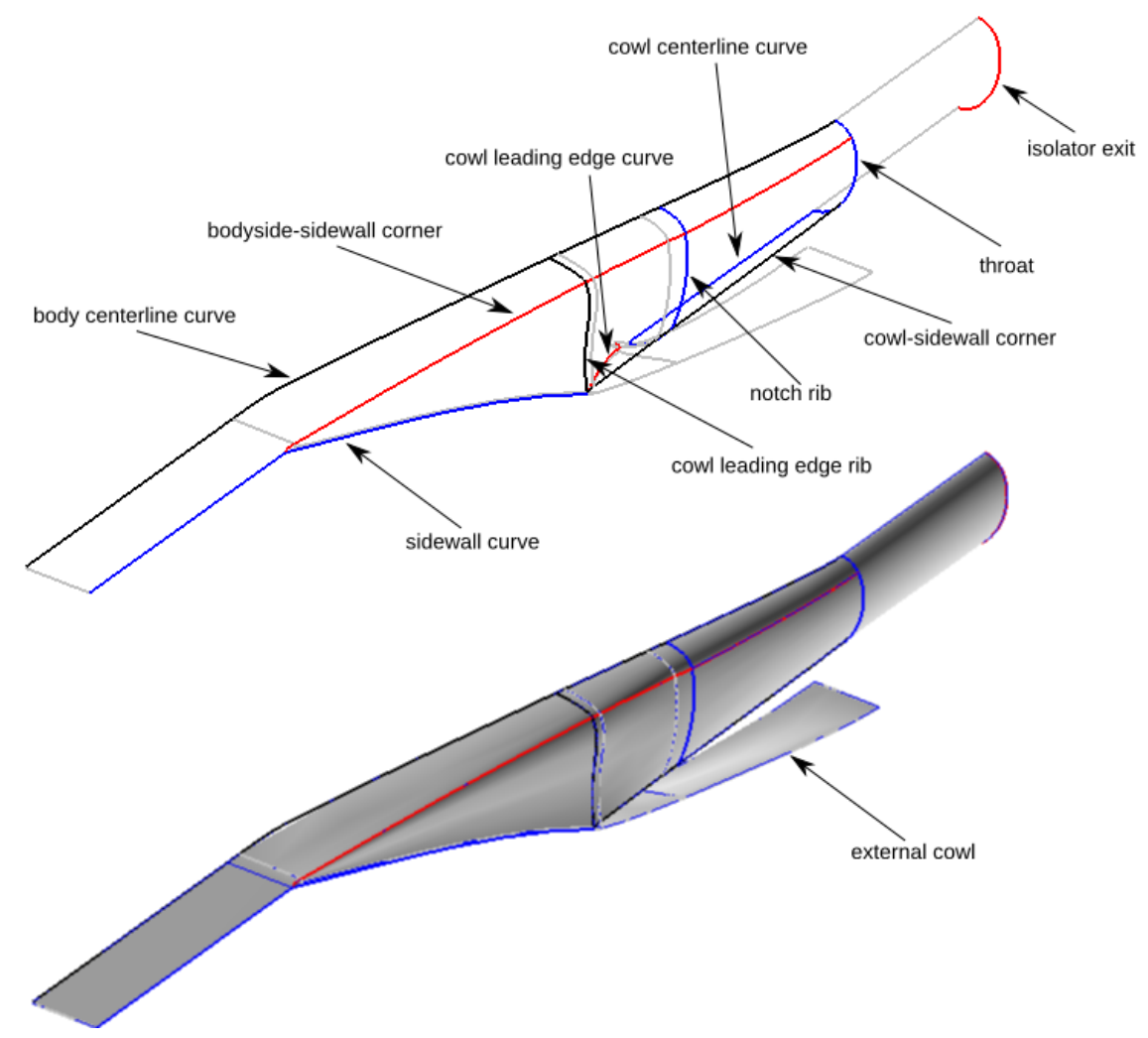

Figure 2. Parametric curves defining a half of a symmetric inlet used in the AML system. Colored curves are controlled by user-defined parameters. Light gray curves are auxiliary curves. Flow is from left to right.

A detailed description of how the parametric geometry is built is given by Ferlemann and Gollan. ${ }^{2}$ The following steps describe how an AML module is used to build the inlet CAD geometry: (1) identify the edges which are sufficient to capture the geometric features of the inlet class; (2) extract a sufficient amount of information from the cloud of points to define the shape of some primary edges; (3) provide extra information (such as leading edge bluntness or isolator length) to generate edges which are not directly available from the cloud of points; (4) build auxiliary edges used in the surface patch definitions; and (5) generate surface patches and stitch them together to form a single surface. This stitching process of surface generation is automated; all that is required is an input file that defines the primary curves and some extra items of information, such as capture width, throat area and leading edge radius. The tool allows flexibility in the number and types of curves used to define edges so that shapes significantly different from the classic REST designs can be created. An initial input file can be generated from the classic design tool. For subsequent design modifications, the designer directly manipulates values in the input deck. The input deck parametrization was carefully chosen to be intuitive to the designer. As values are manipulated the designer gets instant visual feedback on the shape modifications via the geometry display.

\section{Structured grid generation}

A high-fidelity analysis for this design system is defined as solving the three-dimensional Navier-Stokes

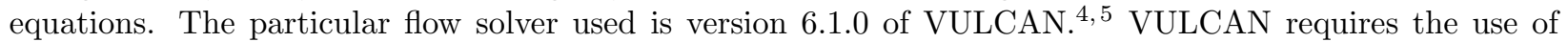
structured grids. The structured volume grids for each design iteration are built using GridPro. ${ }^{6}$ In the GridPro approach to grid generation, the human effort is focused on building an appropriate block topology for the solution volume. During the grid smoothing process, the grid solver projects the appropriate parts of the topology to the surface geometry and moves the topology corners as needed, hence the topology only 
needs to be developed once for a given "class" of geometry. The result is a body-fitted grid which strives to maintain relatively equal cell size and aspect ratio throughout the volume. This would be an appropriate grid for an inviscid calculation. The cell dimensions were roughly 0.01 of an inlet half-width. In order to use this grid for viscous calculations, GridPro provides a utility to cluster additional grid points to any surface. For example, in this work, the initial Euler grids have approximately 2.3 million cells. By contrast, after using the clustering utility, the grids for viscous cases have roughly 4.1 million cells. The GridPro utility was used to give a near wall spacing of $0.0127 \mathrm{~mm}$. This corresponds to $y^{+}$values of less than 2 for most of the model. Previous experience with REST inlet analysis has shown that this order of grid density is sufficient for design purposes.

As the surface changed between each design iteration, it was necessary to move some parts of the GridPro topology. Note, this did not involve starting from scratch at each iteration because the grid topology from a previous design iteration tended to be a good start for each subsequent design iteration. That being said, the manipulation of the wireframe topology remained the most human intensive task (30-60 minutes) in the preparation of structured grids for each design iteration. In the future, this task will also be automated.

In final preparation of the grid, 207 elemental blocks were merged into 13 superblocks. Furthermore, Gridgen $^{7}$ was used in a batch manner for some final tasks: re-orienting blocks, splitting blocks to divide laminar and turbulent regions, and splitting blocks for parallel processing. The total time to generate a new grid (excluding the grid for the initial case) was on the order of an hour.

\section{Inlet flow field analysis}

This section describes the modelling assumptions used to compute the flow fields for each of the design cases. The air was treated as thermally perfect and chemically frozen. The surface boundary condition was set as a no slip, isothermal wall at 400.0 K. A laminar flow assumption was applied up to the end of the sidewall leading edge. Beyond that point, for the remainder of the inlet flow, the $k-\omega$ model $^{8}$ was used to compute turbulent flow with wall-matching functions - this treatment uses wall-matching functions for large values of $y^{+}$and blends towards integrating to the wall as $y^{+}$approaches 1 . Three levels of grid sequencing were used. There were 2000 iterations used at the lower grid resolutions and 6000 iterations for the finest resolution. The convergence of the solutions was determined by the error in mass flow rate across inflow and outflow boundaries. In the worst case, the error for mass flow rate was $0.09 \%$. Typically, the mass flow rate error was less than $0.05 \%$. This level of convergence was considered good enough for design analysis. The inviscid fluxes were computed using the Low Dissipation Flux Split Scheme ${ }^{9}$ and each iteration was advanced using the Diagonalized Approximate Factorization solver. The second order flux reconstruction was turned on after 2000 iterations on the finest grid and this employed a smooth limiter. ${ }^{10}$ In all cases, the incoming flow had no sideslip angle. As such, only half of the inlet geometry was solved by assuming symmetry along the central plane. The computations typically took a little under 15 hours of wall-clock time using 12 processors (3.0 GHz Intel Pentium) of a linux cluster. The solution time can be reduced if more processors were devoted to the simulations. For example, if 180 processors were used, the solution turn-around time would be less than an hour.

\section{Design exercise}

As a demonstration of the toolset for rapid design and analysis of REST-class inlets, an iterative design technique was used to improve certain metrics of the inlet performance. As a design point, vehicle cruise conditions at a Mach number, $M_{0}$, of 8.0 and a dynamic pressure, $q_{0}$, of $48 \mathrm{kPa}$ were selected. The inlet was assumed to be attached to a forebody angled at 6 degrees $\left(\theta_{b}=6^{\circ}\right)$, thus the inlet entrance conditions were taken behind the $6^{\circ}$ bow shock. These conditions are given as State 1 in Table 1. A compression ratio of 20.6 was targetted for the inlet design which corresponds to a throat pressure of approximately $64 \mathrm{kPa}$.

Table 1. Inlet entrance conditions at various free stream Mach numbers

\begin{tabular}{ccccc}
\hline$M_{0}$ & 5.0 & 6.0 & 7.0 & 8.0 \\
\hline$M_{1}$ & 4.39 & 5.18 & 5.93 & 6.64 \\
$p_{1}, \mathrm{kPa}$ & 5517 & 4334 & 3616 & 3112 \\
$T_{1}, \mathrm{~K}$ & 272.8 & 287.6 & 303.1 & 319.3 \\
\hline
\end{tabular}


The throat area was fixed at $1.94 \times 10^{-3} \mathrm{~m}^{2}\left(3 \mathrm{in}^{2}\right)$ for each of the design cases. The total inlet length was not fixed; it varied slightly among cases but was approximately $1 \mathrm{~m}$. In the following section, a baseline design case using the REST design methodology ${ }^{1}$ is presented. As a design exercise, the aim was to reduce the thickness of the boundary layer at the centerline on the bodyside of the inlet. In subsequent sections, an exploratory design stage is conducted by morphing the inlet geometry to effect a reduction on the boundary layer thickness. A step-by-step approach to the design iterations - changing a single geometry aspect per iteration - is presented. The motivation is to give insights about which geometry modifications will yield the greatest change in the metric of interest.

A practical inlet design should be operational over a range of Mach numbers. For this reason, each Mach 8 design case was simulated for off-design inflows corresponding to flight Mach numbers of 5, 6, and 7, all at a dynamic pressure of $48 \mathrm{kPa}$. The inlet entrance conditions corresponding to these flight Mach numbers are given in Table 1. The off-design performance is obtained with minimal extra work.

\section{Classic REST design: baseline case}

As a starting point for the design, an inlet geometry was produced using the classic REST design method. ${ }^{1}$ The underlying compression field used for the streamline-tracing was based on truncating a Busemann diffuser. ${ }^{11}$ The total geometric contraction ratio of the truncated Busemann diffuser was approximately 7.3. When considering inviscid flow through this diffuser at the $M_{1}=6.64$ on-design condition, the compression ratio was 20.5. This was considered close enough to the target compression ratio of 20.6 and was deemed a useful compression field for the inlet design.

For this design exercise, we chose to focus on REST inlets with a 'slender' aspect ratio $(w / h<1)$. The choice of slender inlets was motivated by a Modern Design of Experiments study undertaken by Ferlemann and Gollan ${ }^{2}$ which showed a general trend for better inlet performance at narrower aspect ratios. The capture shape used in the baseline case had an aspect ratio of 0.667 and is shown in Figure 3. Smart ${ }^{1}$ provides details about how the distinctive capture shape for these inlets was formulated. A small blunt leading edge and approximately $25 \mathrm{~cm}$ of length were added to the front of the classic design inlet to develop some boundary layer thickness prior to the initial inlet compression.

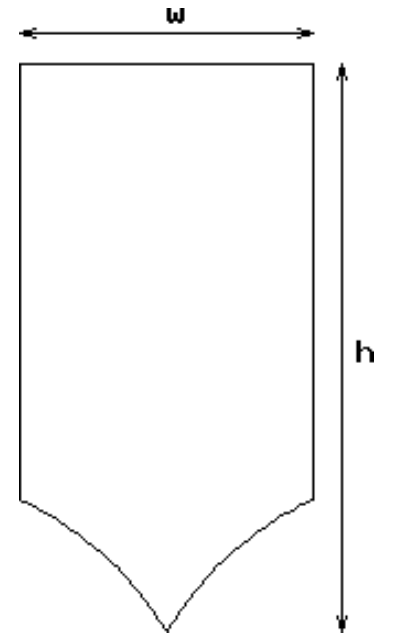

Figure 3. Capture shape used for streamline-tracing in baseline design case. The aspect ratio, $w / h$, is 0.667 .

The results for computing the flow at $M_{0}=8$ for the baseline case (Case 1) are shown in Figures 4 and 5. In Figure 4, contours of Mach number are displayed: the top image shows the contours along the symmetry plane of the inlet; the bottom image shows again the contours along the symmetry plane along with cuts at four longitudinal locations (sidewall end, notch, throat and isolator exit). The Mach number contours show that the shock-on-lip design intent is achieved in this inlet. It may also be noted that a small region of separation occurs on the bodyside, just upstream of the throat. The onset of separation is difficult to predict with simple methods utilized during the design stage. This gives further motivation to using high-fidelity tools early so that the designer can quickly identify potential problems at the beginning of the design process. The pressure distribution along the inlet surface is shown in Figure 5. 


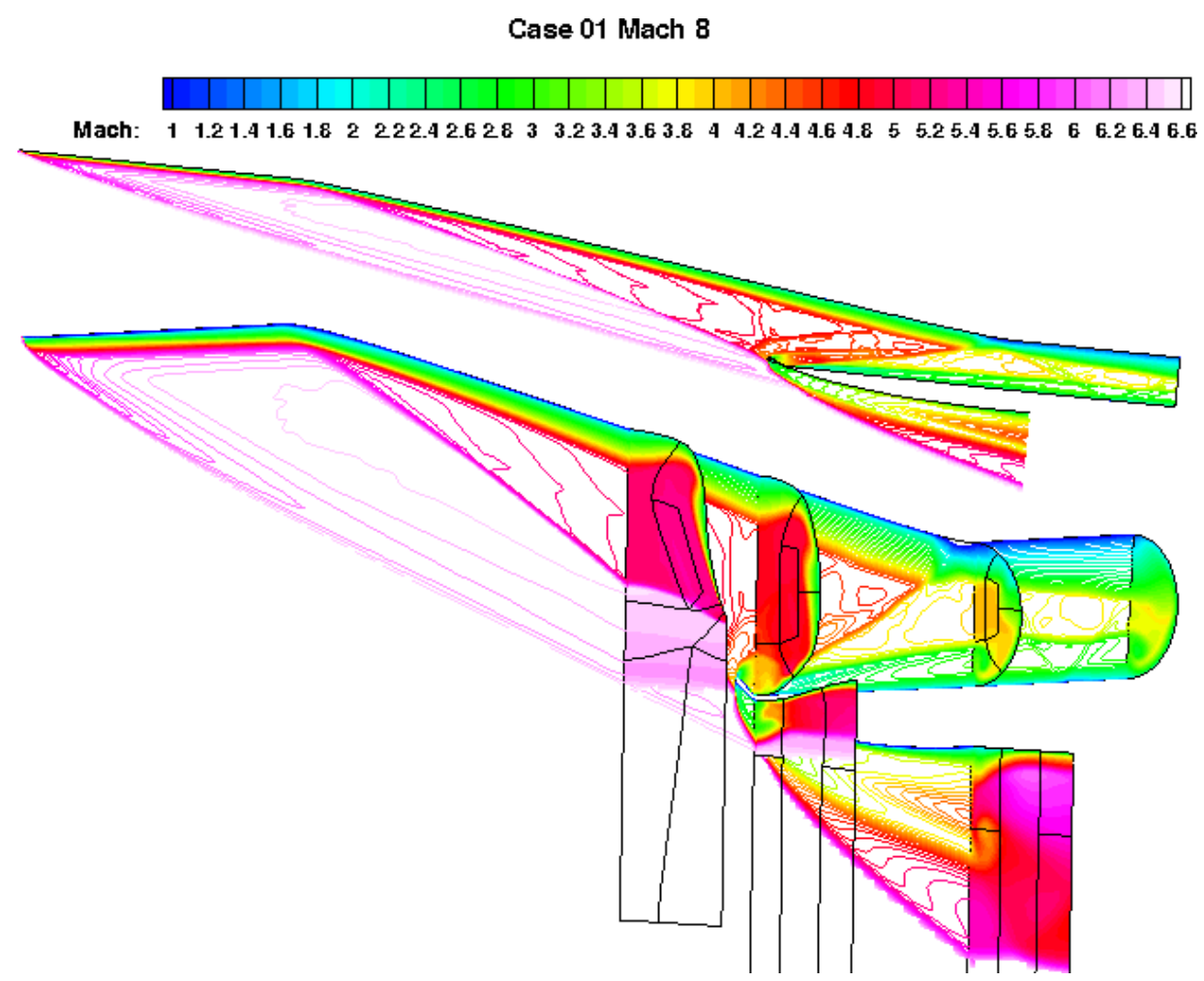

Figure 4. Contours of Mach number for the baseline case (Case 1) at $M_{0}=8$. Top image is a cut at the symmetry plane. Bottom image shows a perspective view with cuts at the end of sidewall, notch, throat and isolator exit. Flow is from left to right. The black lines in the cross-plane views show block edges from the grid.

A number of inlet metrics are given in Table 2. The results for this baseline case are listed under Case 1. No single performance metric provides the designer with a sufficient amount of information for assessment. Integrated and local metrics are useful. Selected integrated metrics include: total drag (including external cowl), throat stream thrust per unit mass capture $(F / \dot{m})$, total pressure recovery, and boundary layer area ratio - the ratio of the throat area consumed by the boundary layer compared to the total throat area (boundary layer edge is defined where $99.5 \%$ of free stream total enthalpy is recovered). Selected local metrics included wall shear stress around the perimeter (minimum, average, maximum) and boundary layer thickness at the body centerline. The wall shear stress was computed since it can be used in correlations for estimating back-pressure capability. The boundary layer thicknesses at the body centerline were selected, as opposed to some other location on the perimeter, because that tends to be the location of greatest thickness or 'weakest' boundary layer flow. All integrated values across an area used a flux-conserved technique. ${ }^{12}$ The one-dimensional pressure at the throat was computed as $67.2 \mathrm{kPa}$. This was close enough to the target value of $64 \mathrm{kPa}$ that no further iteration was deemed necessary. Of particular note is the value for boundary layer thickness on the body centerline, $\delta=15.0 \mathrm{~mm}$. The objective was to reduce this value in subsequent design iterations.

Contour plots of Mach number for free stream Mach numbers of 7, 6, and 5 are shown in Figures 6, 7, and 8 respectively. The Mach 5 and 6 simulations indicate additional flow separation in the isolator along the body centerline even with no combustor back-pressure. The inlet metrics for the off-design Mach numbers are given Tables 3, 4 and 5. Some general trends are noted as the Mach number decreases: the mass capture remains nearly constant; $F / \dot{m}$ decreases; total pressure recovery increases; minimum wall shear stress decreases; the boundary layer area ratio, $A_{\delta} / A_{c}$, increases; and the boundary layer thickness, $\delta$, increases, which indicates greater adverse viscous effects at off-design Mach numbers. 


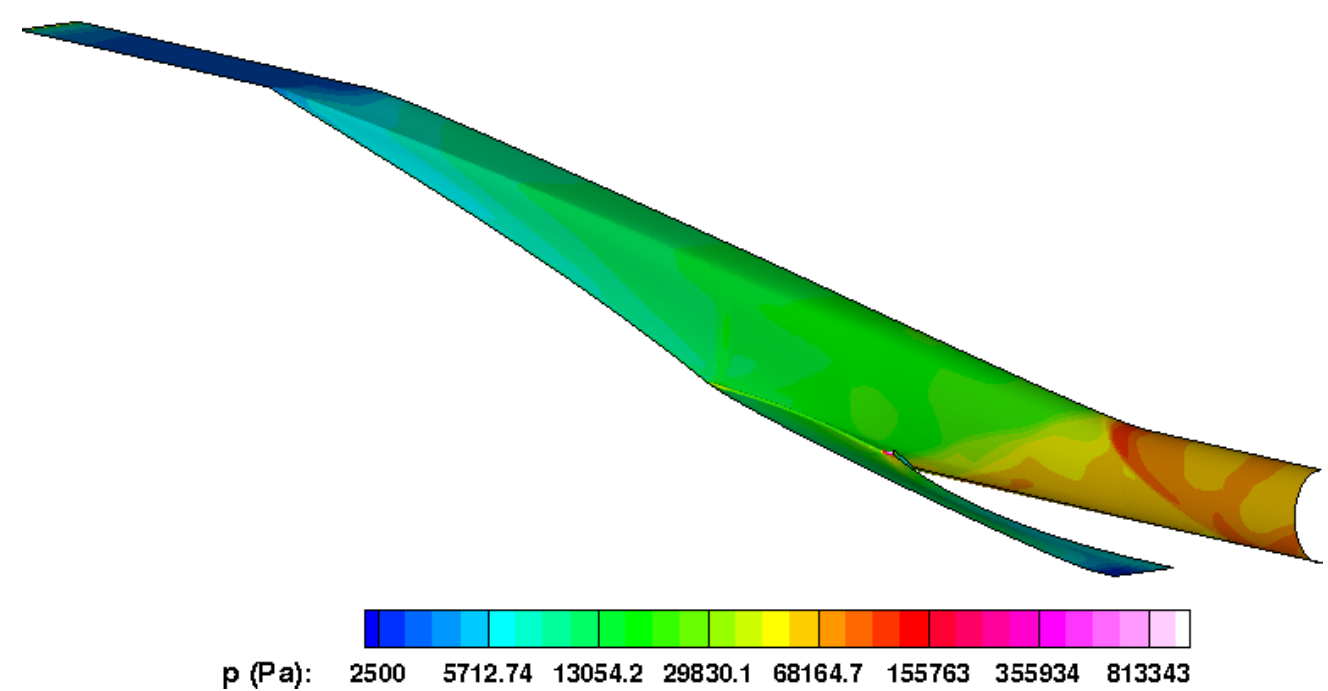

Figure 5. Contours of pressure displayed on the inlet surface for the baseline case with inflow corresponding to $M_{0}=8$. Flow is from left to right.

Table 2. Results at Mach 8: Cases 1, 3-8 ${ }^{a}$

\begin{tabular}{|c|c|c|c|c|c|c|c|}
\hline Metric & Case 1 & Case 3 & Case 4 & Case 5 & Case 6 & Case 7 & Case 8 \\
\hline$\dot{m}, \mathrm{~kg} / \mathrm{s}$ & 0.476 & 0.475 & 0.458 & 0.469 & 0.469 & 0.474 & 0.475 \\
\hline$D, \mathrm{~N}$ & 230 & 210 & 190 & 229 & 229 & 227 & 222 \\
\hline$A, \mathrm{~m}^{2}$ & 0.124 & 0.135 & 0.138 & 0.124 & 0.124 & 0.129 & 0.130 \\
\hline \multicolumn{8}{|c|}{ One-dimensionalized values at throat } \\
\hline$F, \mathrm{~N}$ & 1012 & 1015 & 981 & 997 & 996 & 1011 & 995 \\
\hline$F / \dot{m}, \mathrm{~N} . \mathrm{s} / \mathrm{kg}$ & 2125 & 2137 & 2142 & 2125 & 2125 & 2130 & 2095 \\
\hline$p, \mathrm{kPa}$ & 67.2 & 66.2 & 63.5 & 66.1 & 66.0 & 66.1 & 73.6 \\
\hline$M$ & 3.31 & 3.33 & 3.34 & 3.31 & 3.32 & 3.34 & 3.13 \\
\hline$p_{t}$ recovery & 0.288 & 0.295 & 0.286 & 0.284 & 0.285 & 0.295 & 0.236 \\
\hline \multicolumn{8}{|c|}{ Values on the perimeter at throat } \\
\hline$\tau_{m i n}, \mathrm{~Pa}$ & 1052 & 593 & 290 & 637 & 622 & 514 & 467 \\
\hline$\tau_{\text {avg }}, \mathrm{Pa}$ & 1361 & 1407 & 1241 & 1457 & 1458 & 1421 & 1513 \\
\hline$\tau_{\max }, \mathrm{Pa}$ & 1601 & 2213 & 2089 & 1918 & 1939 & 2318 & 2014 \\
\hline$\tau_{\max }-\tau_{\min }, \mathrm{Pa}$ & 549 & 1620 & 1799 & 1281 & 1317 & 1804 & 1547 \\
\hline$A_{\delta} / A_{c}$ & 0.533 & 0.588 & 0.587 & 0.523 & 0.523 & 0.611 & 0.609 \\
\hline \multicolumn{8}{|c|}{ Values on the body centerline at throat } \\
\hline$\delta, \mathrm{mm}$ & 15.02 & 15.95 & 11.76 & 13.56 & 13.86 & 18.28 & 12.59 \\
\hline$\delta^{*}, \mathrm{~mm}$ & 6.56 & 8.81 & 6.22 & 6.28 & 6.35 & 7.73 & 6.72 \\
\hline$\theta, \mathrm{mm}$ & 1.99 & 1.60 & 1.07 & 1.75 & 1.81 & 1.90 & 1.58 \\
\hline
\end{tabular}

${ }^{a}$ Case 2 is omitted because its values at the throat cannot be fairly compared to the other cases.

The design intent for Case 2 only considered geometry changes up to the end of the sidewall. 


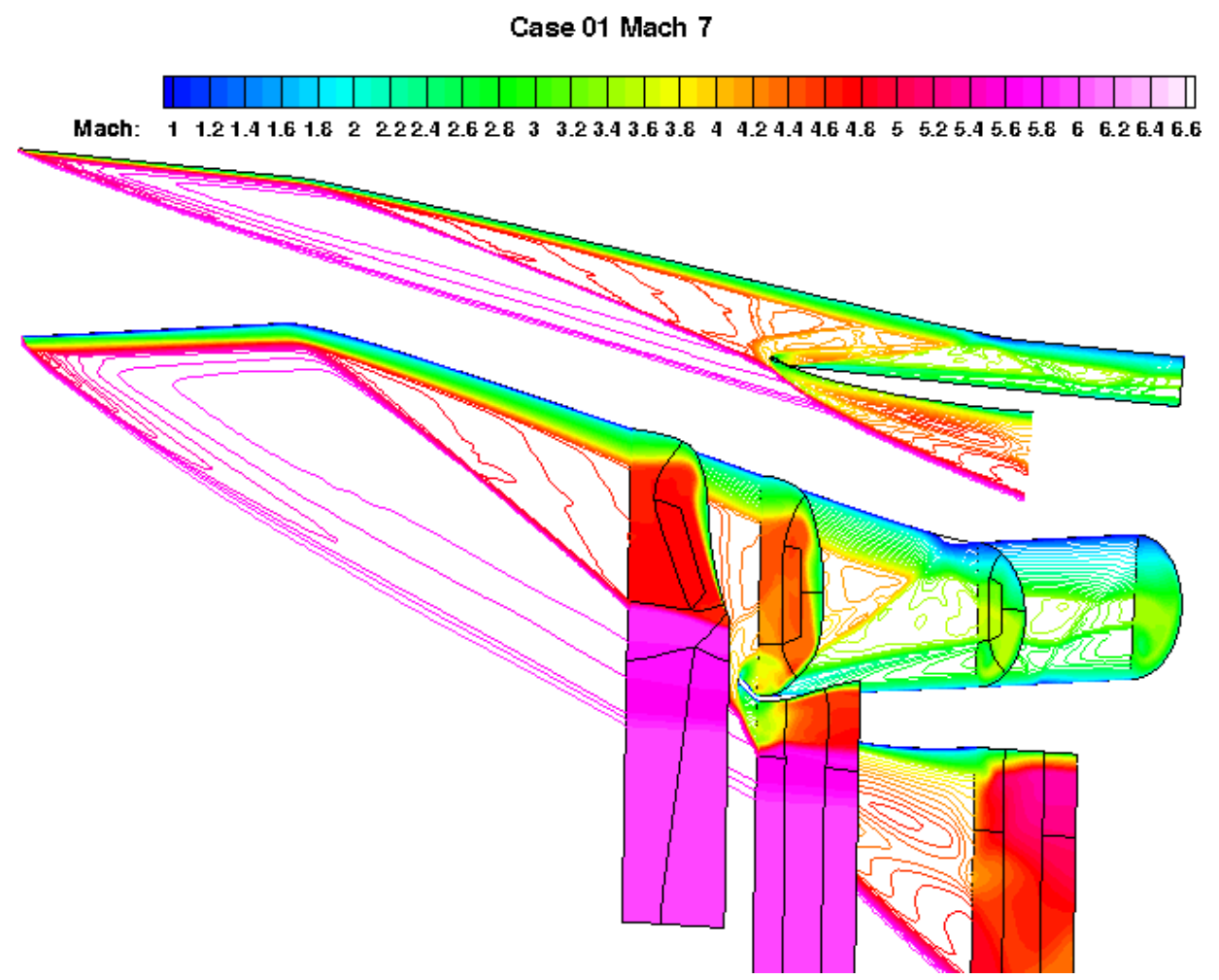

Figure 6. Contours of Mach number for Case 1 with $M_{0}=7$. Flow is from left to right.

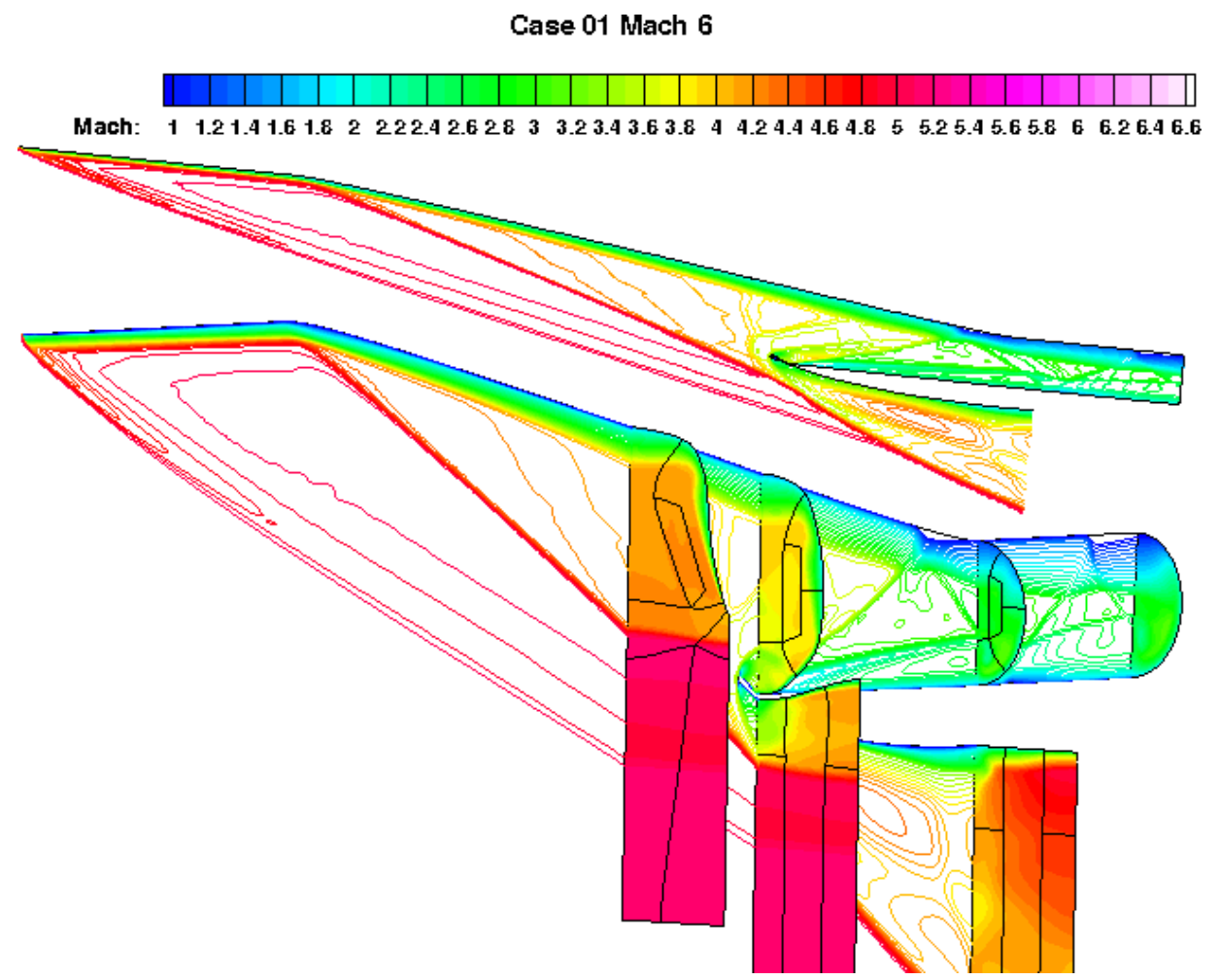

Figure 7. Contours of Mach number for Case 1 with $M_{0}=6$. Flow is from left to right. 


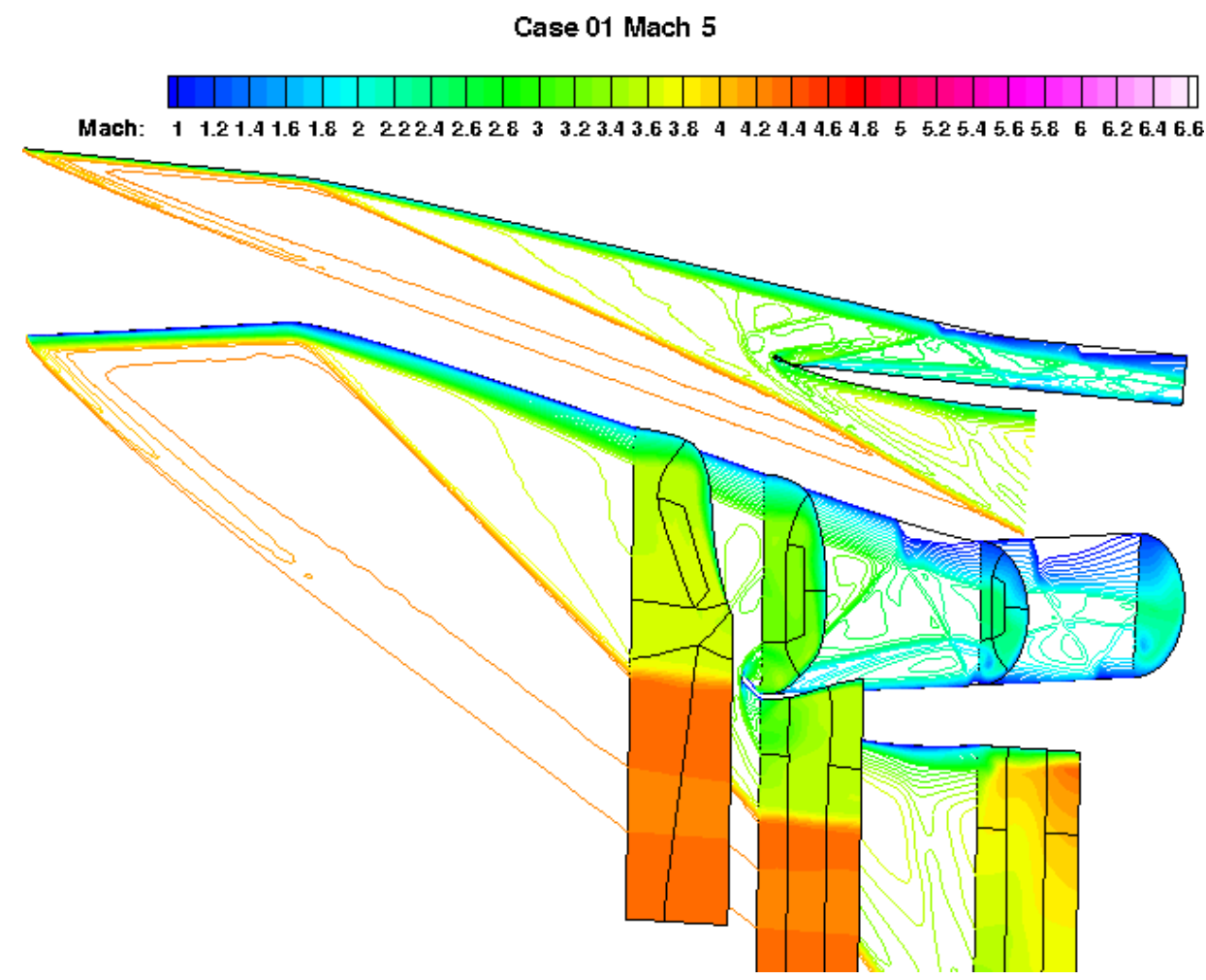

Figure 8. Contours of Mach number for Case 1 with $M_{0}=5$. Flow is from left to right.

Table 3. Results at Mach 7: Cases 1, 3-8

\begin{tabular}{|c|c|c|c|c|c|c|c|}
\hline Metric & Case 1 & Case 3 & Case 4 & Case 5 & Case 6 & Case 7 & Case 8 \\
\hline$\dot{m}, \mathrm{~kg} / \mathrm{s}$ & 0.479 & 0.488 & 0.471 & 0.469 & 0.469 & 0.487 & 0.492 \\
\hline$D, \mathrm{~N}$ & 229 & 214 & 193 & 228 & 228 & 228 & 224 \\
\hline$A, \mathrm{~m}^{2}$ & 0.124 & 0.135 & 0.138 & 0.124 & 0.124 & 0.129 & 0.130 \\
\hline \multicolumn{8}{|c|}{ One-dimensionalized values at throat } \\
\hline$F, \mathrm{~N}$ & 874 & 898 & 871 & 856 & 855 & 892 & 885 \\
\hline$F / \dot{m}, \mathrm{~N} . \mathrm{s} / \mathrm{kg}$ & 1825 & 1840 & 1849 & 1824 & 1825 & 1833 & 1801 \\
\hline$p, \mathrm{kPa}$ & 71.8 & 72.5 & 69.4 & 70.3 & 70.1 & 71.9 & 79.8 \\
\hline$M$ & 2.95 & 2.97 & 2.98 & 2.95 & 2.95 & 2.98 & 2.82 \\
\hline$p_{t}$ recovery & 0.347 & 0.364 & 0.353 & 0.341 & 0.341 & 0.364 & 0.309 \\
\hline \multicolumn{8}{|c|}{ Values on the perimeter at throat } \\
\hline$\tau_{m i n}, \mathrm{~Pa}$ & 992 & 607 & 593 & 670 & 663 & 674 & 595 \\
\hline$\tau_{a v g}, \mathrm{~Pa}$ & 1254 & 1151 & 1124 & 1228 & 1229 & 1239 & 1378 \\
\hline$\tau_{\max }, \mathrm{Pa}$ & 1605 & 1634 & 1744 & 1578 & 1615 & 1706 & 2793 \\
\hline$\tau_{\max }-\tau_{\min }, \mathrm{Pa}$ & 613 & 1027 & 1151 & 908 & 952 & 1032 & 2198 \\
\hline$A_{\delta} / A_{c}$ & 0.538 & 0.583 & 0.552 & 0.531 & 0.533 & 0.558 & 0.590 \\
\hline \multicolumn{8}{|c|}{ Values on the body centerline at throat } \\
\hline$\delta, \mathrm{mm}$ & 16.77 & 17.02 & 11.07 & 16.16 & 16.21 & 17.37 & 12.01 \\
\hline$\delta^{*}, \mathrm{~mm}$ & 7.94 & 8.59 & 5.08 & 7.72 & 7.87 & 6.95 & 5.67 \\
\hline$\theta, \mathrm{mm}$ & 1.89 & 1.68 & 1.30 & 1.83 & 1.83 & 2.08 & 1.32 \\
\hline
\end{tabular}


Table 4. Results at Mach 6: Cases 1, 3-8

\begin{tabular}{lccccccc}
\hline Metric & Case 1 & Case 3 & Case 4 & Case 5 & Case 6 & Case 7 & Case 8 \\
\hline$\dot{m}, \mathrm{~kg} / \mathrm{s}$ & 0.476 & 0.493 & 0.481 & 0.464 & 0.463 & 0.486 & 0.491 \\
$D, \mathrm{~N}$ & 237 & 222 & 201 & 236 & 235 & 235 & 224 \\
$A, \mathrm{~m}^{2}$ & 0.124 & 0.135 & 0.138 & 0.124 & 0.124 & 0.129 & 0.130 \\
\hline One-dimensionalized values & at throat & & & & & \\
$F, \mathrm{~N}$ & 727 & 759 & 744 & 709 & 708 & 744 & 741 \\
$F / \dot{m}, \mathrm{~N} . \mathrm{s} / \mathrm{kg}$ & 1528 & 1540 & 1547 & 1527 & 1527 & 1533 & 1509 \\
$p, \mathrm{kPa}$ & 77.7 & 81.3 & 78.9 & 75.4 & 75.4 & 79.0 & 87.7 \\
$M$ & 2.56 & 2.55 & 2.54 & 2.56 & 2.56 & 2.56 & 2.41 \\
$p_{t} \mathrm{recovery}$ & 0.421 & 0.434 & 0.420 & 0.413 & 0.413 & 0.431 & 0.374 \\
\hline Values on the perimeter at & throat & & & & & \\
$\tau_{\text {min }}, \mathrm{Pa}$ & 682 & 531 & 579 & 553 & 546 & 531 & 403 \\
$\tau_{\text {avg }}, \mathrm{Pa}$ & 949 & 1020 & 986 & 982 & 981 & 1011 & 1286 \\
$\tau_{\text {max }}, \mathrm{Pa}$ & 1373 & 1510 & 1393 & 1470 & 1470 & 1356 & 2072 \\
$\tau_{\text {max }}-\tau_{\text {min }}, \mathrm{Pa}$ & 691 & 979 & 814 & 917 & 924 & 825 & 1669 \\
$A_{\delta} / A_{c}$ & 0.577 & 0.587 & 0.555 & 0.578 & 0.575 & 0.570 & 0.621 \\
\hline Values on the body & centerline at throat & & & & \\
$\delta, \mathrm{mm}$ & 18.17 & 18.57 & 11.46 & 17.99 & 17.97 & 18.67 & 9.71 \\
$\delta^{*}, \mathrm{~mm}$ & 8.06 & 8.66 & 5.38 & 8.19 & 8.26 & 8.62 & 3.90 \\
$\theta, \mathrm{mm}$ & 2.05 & 1.93 & 1.32 & 2.04 & 2.07 & 3.00 & 1.05 \\
\hline
\end{tabular}

Table 5. Results at Mach 5: Cases 1, 3-8

\begin{tabular}{|c|c|c|c|c|c|c|c|}
\hline Metric & Case 1 & Case 3 & Case 4 & Case 5 & Case 6 & Case 7 & Case 8 \\
\hline$\dot{m}, \mathrm{~kg} / \mathrm{s}$ & 0.475 & 0.497 & 0.491 & 0.462 & 0.461 & 0.489 & 0.496 \\
\hline$D, \mathrm{~N}$ & 247 & 235 & 216 & 245 & 245 & 248 & 238 \\
\hline$A, \mathrm{~m}^{2}$ & 0.124 & 0.135 & 0.138 & 0.124 & 0.124 & 0.129 & 0.130 \\
\hline \multicolumn{8}{|c|}{ One-dimensionalized values at throat } \\
\hline$F, \mathrm{~N}$ & 585 & 619 & 610 & 570 & 570 & 602 & 606 \\
\hline$F / \dot{m}$, N.s $/ \mathrm{kg}$ & 1232 & 1245 & 1242 & 1235 & 1235 & 1230 & 1222 \\
\hline$p, \mathrm{kPa}$ & 89.9 & 98.5 & 96.6 & 86.6 & 86.6 & 94.6 & 106.3 \\
\hline$M$ & 2.08 & 2.02 & 2.02 & 2.09 & 2.09 & 2.05 & 1.91 \\
\hline$p_{t}$ recovery & 0.507 & 0.501 & 0.496 & 0.499 & 0.497 & 0.508 & 0.452 \\
\hline \multicolumn{8}{|c|}{ Values on the perimeter at throat } \\
\hline$\tau_{\min }, \mathrm{Pa}$ & 465 & 407 & 427 & 196 & 229 & 420 & -101 \\
\hline$\tau_{a v g}, \mathrm{~Pa}$ & 641 & 834 & 786 & 752 & 753 & 779 & 861 \\
\hline$\tau_{\max }, \mathrm{Pa}$ & 908 & 1310 & 1317 & 1109 & 1101 & 1089 & 1477 \\
\hline$\tau_{\max }-\tau_{\min }, \mathrm{Pa}$ & 443 & 903 & 890 & 913 & 872 & 669 & 1376 \\
\hline$A_{\delta} / A_{c}$ & 0.584 & 0.634 & 0.591 & 0.583 & 0.579 & 0.600 & 0.651 \\
\hline \multicolumn{8}{|c|}{ Values on the body centerline at throat } \\
\hline$\delta, \mathrm{mm}$ & 19.09 & 20.47 & 12.57 & 19.48 & 19.48 & 20.73 & 11.80 \\
\hline$\delta^{*}, \mathrm{~mm}$ & 7.92 & 9.07 & 5.74 & 8.40 & 8.51 & 9.11 & 4.59 \\
\hline$\theta, \mathrm{mm}$ & 2.37 & 2.16 & 1.47 & 2.42 & 2.45 & 2.36 & 1.54 \\
\hline
\end{tabular}




\section{Exploratory design iterations}

In the first stage of the study, some intuitive shape changes were made and the performance was assessed via a full Navier-Stokes CFD calculation. The idea was to manually improve the inlet flow by making progressive changes to the geometry beginning from the inlet capture and working downstream towards the throat. The nature of supersonic flow in the inlet meant that any desirable changes made upstream should not be affected by later geometry changes downstream. The following paragraphs describe the intuition that was used to drive the geometry changes. The nomenclature related to the specific geometry changes may be found by referring to Figure 2. Certain aspects of the parametrized geometry are tightly coupled and so minor adjustments were required in some of the other controlling curves in order to construct a well-formed surface. Only the major geometry changes are reported here.

\section{Changes from Case 1 to Case 2}

One common aspect of the inlet geometry based on the classic design method is that the sidewall compression begins earlier than the bodyside compression. This contributes to the boundary layer build-up toward the centerline of the bodyside. Thus, the first line of thinking was to begin the bodyside compression earlier in an attempt to somewhat equalize the sidewall compression. There were two primary geometry changes made to help achieve the equalized compression: (1) the curve controlling the bodyside centerline was moved forward, so as to begin compressing the flow sooner, and (2) the corner line where the body meets the sidewall was pulled out, so as to reduce the aggressiveness of the corner compression. Figure 9 depicts the changes in geometry between the baseline case and Case 2 - the labels correspond to the changes discussed in the text. Most of the changes are subtle. The most distinct change is visible in the corner at the location where the sidewall ends: the new surface has been pulled out with respect to the original surface.

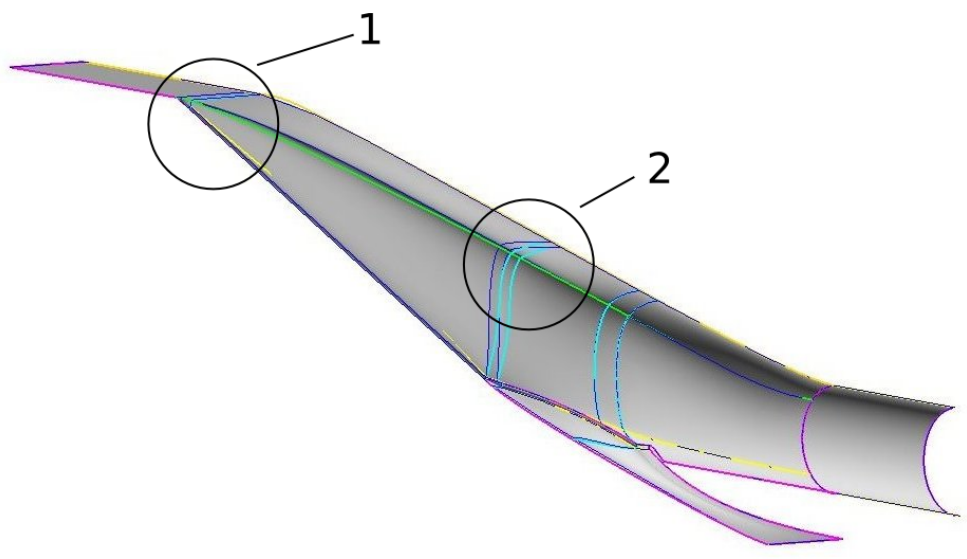

Figure 9. Geometry changes from Case 1 to Case 2. The new geometry, Case 2, is shown by the surface. The non-dark blue curves represent the control geometry for the previous iteration, Case 1 . Flow is from left to right.

The objective of Case 2 was to reduce the boundary layer thickness only so far as the end of the sidewall (where the internal cowl begins). This was achieved: in Case 1, the boundary layer thickness on the body centerline at the location where the sidewall ends is $14.0 \mathrm{~mm}$, in Case 2, $11.4 \mathrm{~mm}$. Note that the Case 2 results have been omitted from the tables of results because it is unfair to compare the results at the throat when the focus was the end of the sidewall for this first iteration. The contours of Mach number for this case at the on-design condition are shown in Figure 10.

\section{Changes from Case 2 to Case 3}

In keeping with the thinking of trying to equalize the compression amongst the surfaces, in this iteration an attempt was made to reduce the amount of sidewall compression (as opposed to increasing the compression on the bodyside surface as in the previous case). To explore this idea, the aspect ratio of the throat was increased from 0.667 (which gives an ellipse which is taller than it is wide) to 1.0 (a circle). This is labelled as (1) in Figure 11. 


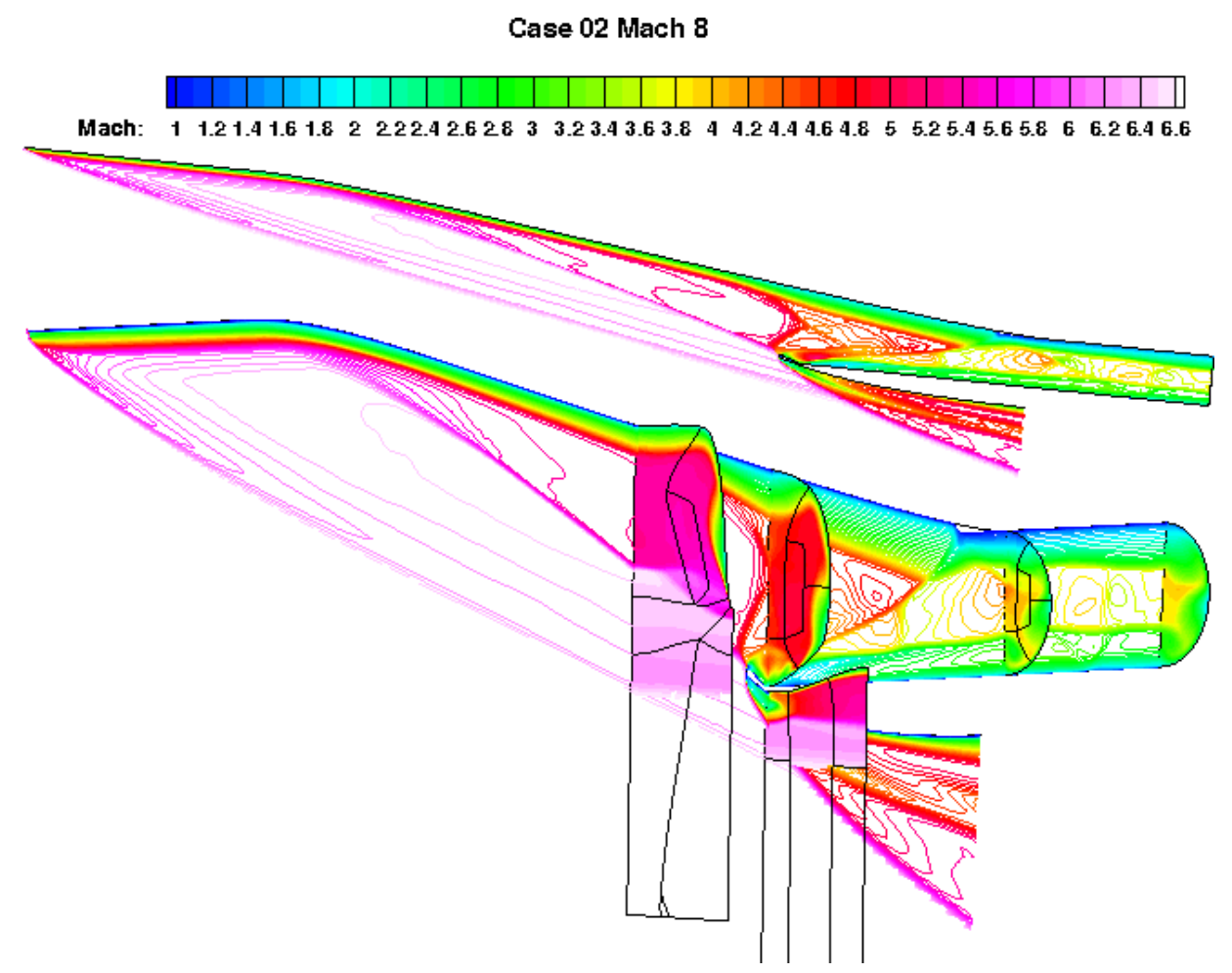

Figure 10. Contours of Mach number for Case 2 with $M_{0}=8$. Flow is from left to right.

The other primary change in this iteration was to cant the throat and isolator back in line with the waterline (label (2) in Figure 11). In scramjet engine designs which make use of a REST inlet, it is common to take a 'turn' somewhere in the engine in order to align the thrust with the vehicle direction. However, it is unclear where best to place that turn: it would require a study of a vehicle-integrated REST-based engine to properly determine the best place. It is also likely that the best place is dependent upon larger vehicle configuration choices and the mission profile. In any case, the benefit or otherwise of placing the turn at the throat was investigated; the parametrized geometry allows that change to be easily made. A comparison of geometry changes between Case 2 and Case 3 is shown in Figure 11.

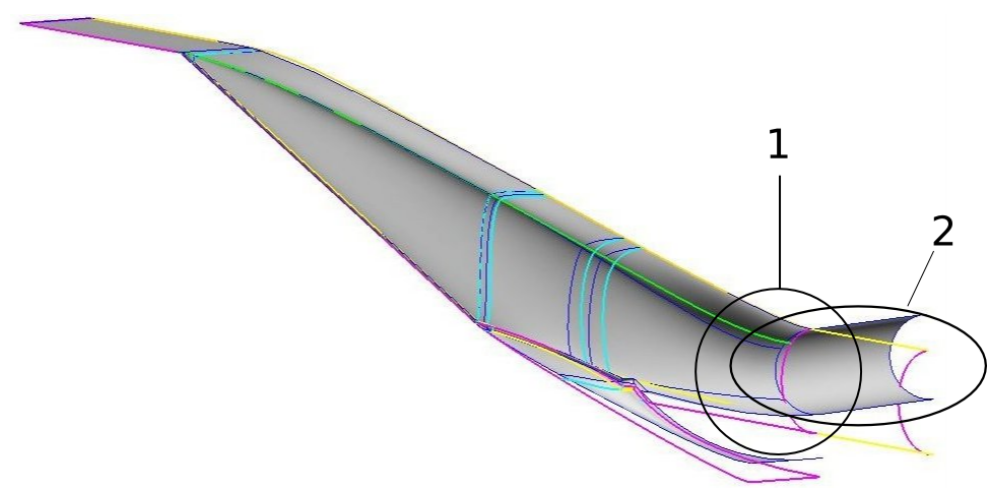

Figure 11. Geometry changes from Case 2 to Case 3. The new geometry, Case 3, is shown by the surface. The non-dark blue curves represent the geometry for the previous iteration, Case 2. Flow is from left to right.

Contours of Mach number at $M_{0}=8$ are shown in Figure 12, which can be compared to the baseline case in Figure 4. The corresponding performance metrics are listed in Table 2. Compared to the baseline case, the total drag, $F / \dot{m}$, and total pressure recovery have improved; while the minimum shear stress, the boundary layer area ratio, and the boundary layer thickness have not improved. The same trend held at 
each of the off-design Mach numbers (Tables 3-5). Although not tabulated, it is interesting to note, based on the core Mach number at the end of the isolator, that the baseline case exhibits greater losses through the isolator than Case 3. Therefore, canting the isolator back parallel to the vehicle waterline is a viable option.

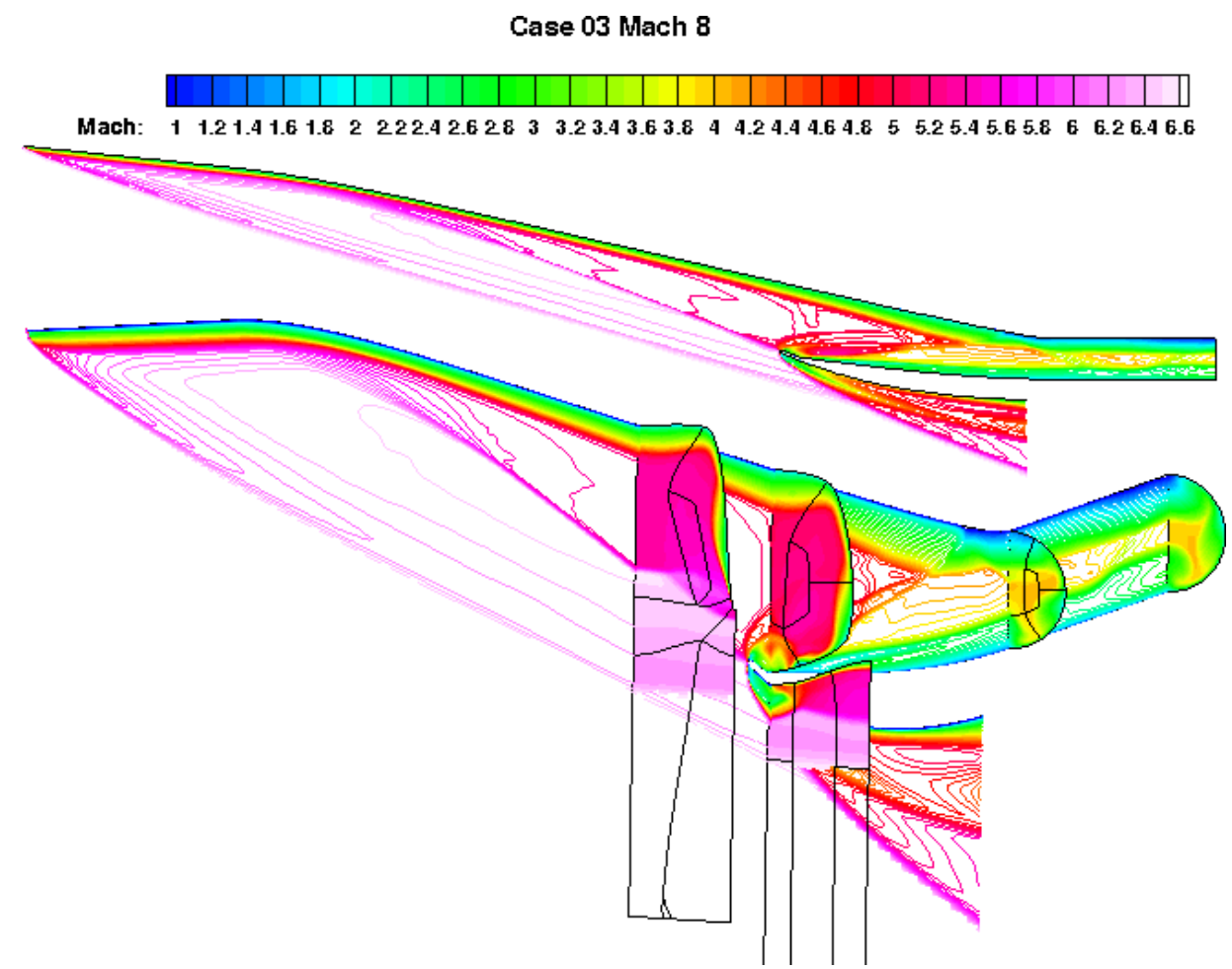

Figure 12. Contours of Mach number for Case 3 with $M_{0}=8$. Flow is from left to right.

\section{Changes from Case 3 to Case 4}

Although the change to a circular throat did not further reduce the boundary layer thickness in the previous iteration, it was decided to continue the experiment with aspect ratio in Case 4 by increasing the value to 1.75. In effect, this changes the orientation of the ellipse at the throat from the original design (denoted with label (1) in Figure 13). The other major geometry change in this iteration was the introduction of a bump along the bodyside centerline, shown with label (2) in Figure 13. The bump was added to explore the idea of pushing the boundary layer away from the bodyside centerline. As in previous cases, a number of small adjustments were required in other controlling curves to accommodate the primary geometry changes. Figure 13 shows the changes in geometry between Cases 3 and 4 .

Contours of Mach number at the on-design condition are shown in Figure 14 and performance metrics are given in Table 2. In this case there is a modest reduction in boundary layer thickness, down to $11.8 \mathrm{~mm}$, while most other metrics are comparable to previous cases. In comparison to the baseline case, the boundary layer thickness has been reduced by about $20 \%$ of its original value. Compared to Case 3, the total drag, $F / \dot{m}$ and boundary layer thickness have improved; while total pressure recovery and minimum shear stress have not improved. This is viewed as a pleasing result: we have demonstrated that it is possible to make design modifications within our system and positively influence a certain metric of performance.

\section{Step-by-step design iterations}

In the second part of the study, we took the lessons learned about the effect of geometry changes in the first part and applied the changes systematically, one change at a time. The hope was to identify which shape changes were the biggest drivers in terms of influencing the boundary layer growth on the bodyside surface. The individual changes were applied in sequence to the baseline inlet geometry and the flow field was analyzed; the subsequent sections present these changes and the results. 


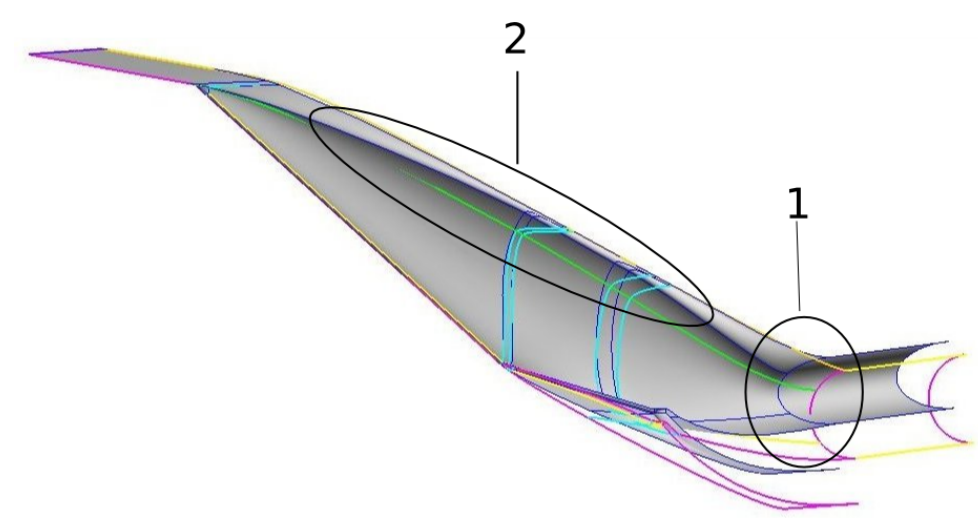

Figure 13. Geometry changes from Case 3 to Case 4. The new geometry, Case 4, is shown by the surface. The non-dark blue curves represent the geometry for the previous iteration, Case 3. Flow is from left to right.

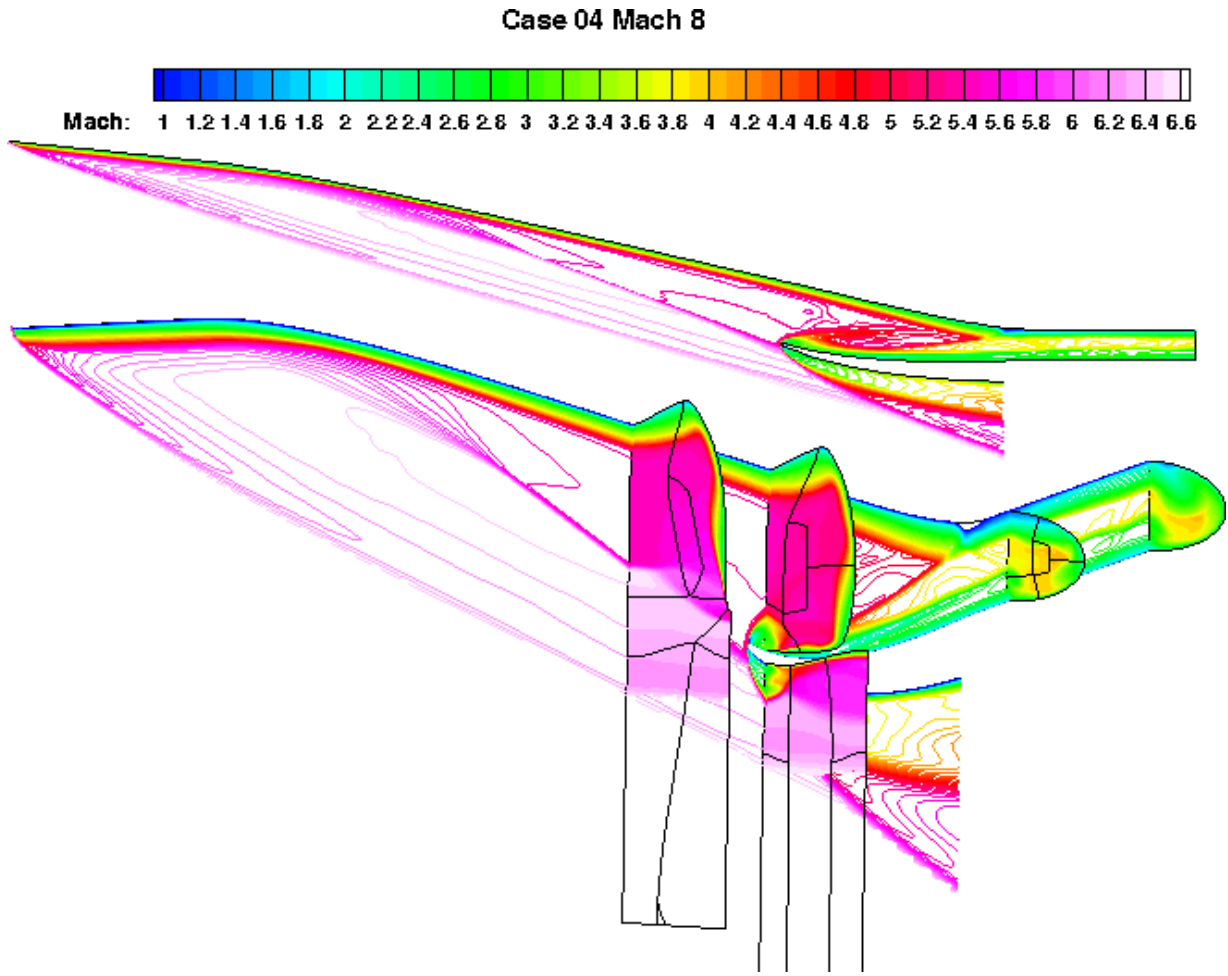

Figure 14. Contours of Mach number for Case 4 with $M_{0}=8$. Flow is from left to right.

\section{Changes from Case 1 to Case 5}

The swept leading edges of a REST inlet have a distinctive shape which comes from starting the edges at the intersection of the capture shape and the conical shock front which is part of the underlying axisymmetric compression field used for streamline tracing. The specific shape of the leading edges is considered important to correctly generate the primary shock structure of the underlying compression field. It is interesting then, in the first design iteration, to change the sidewall leading edge to a straight line, which would reduce manufacturing complexity, and assess the effect on the flow field. In this first iteration, this is strictly the only change that has been made to the inlet shape: the curved sidewall leading edge has been replaced with a straight line.

The inlet performance metrics for Case 5 are listed in Table 2. In general, the straightened sidewall has had little effect on the global metrics. The most noticeable local changes are to the shear stress distributions at the throat. The large influence locally is likely due to small variations in the shock location. In comparing 
the flow field shown in Figure 4 for Case 1 to that shown in Figure 15 for Case 5, it is difficult to discern much change in bulk flow features. The boundary layer thickness on the body centerline was reduced to $13.6 \mathrm{~mm}$ from $15.0 \mathrm{~mm}$ in Case 1.

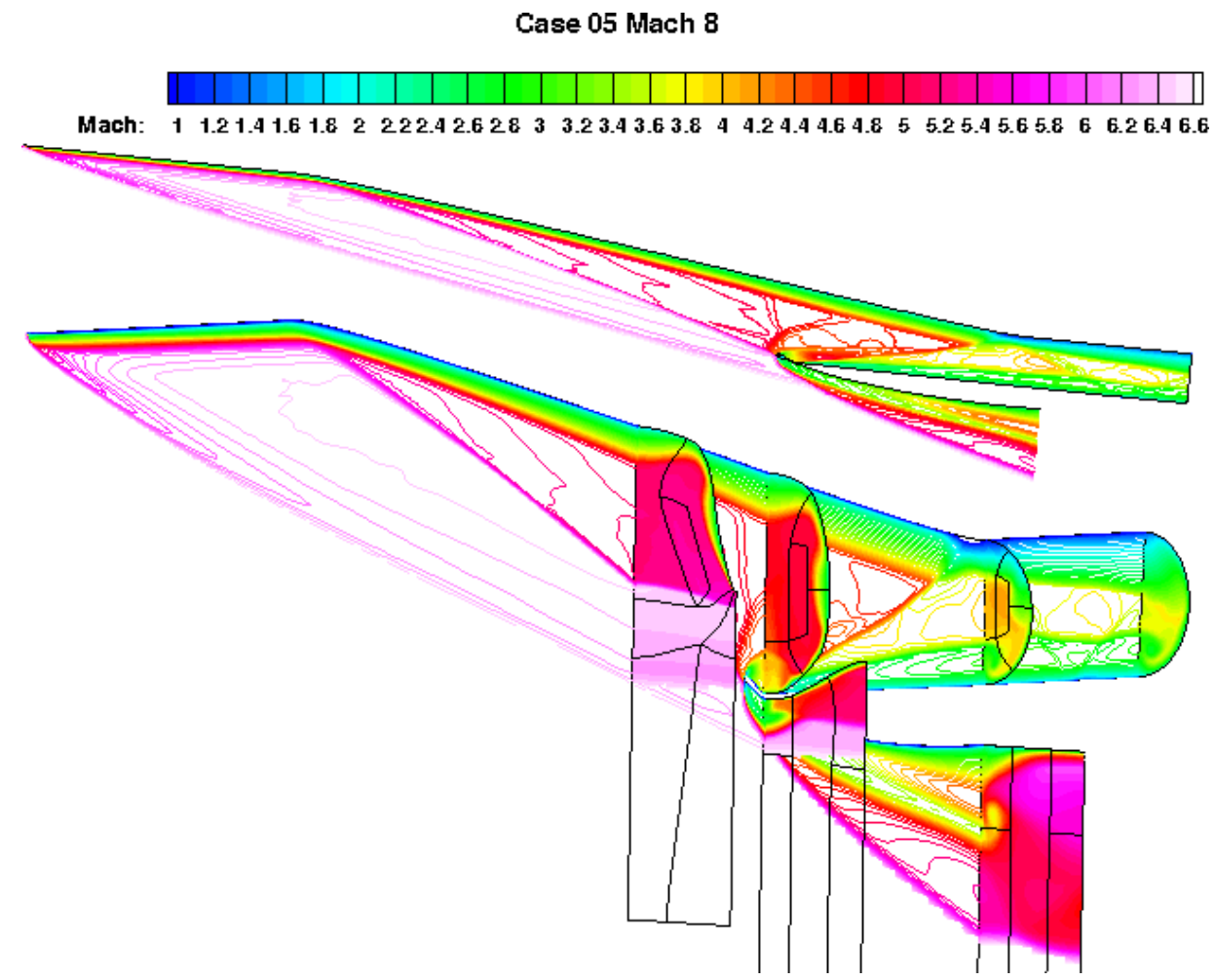

Figure 15. Contours of Mach number for Case 5 with $M_{0}=8$. Flow is from left to right.

\section{Changes from Case 5 to Case 6}

In the second design change, the body centerline was moved forward by about $7 \%$ of its original total length. This has the effect of lengthening the entire bodyside surface and begins the bodyside compression earlier than in the baseline case. Each design iteration includes the changes from the previous iteration. So, in this case, the straightened sidewall still remains.

The results of the flow field analysis for Case 6 are shown in Table 2 and a visualization of the Mach number contours are displayed in Figure 16. Again, most of the metrics for inlet performance are comparable with those from the previous iterations. Interestingly, the boundary layer thickness, $\delta$, has actually increased slightly to $13.9 \mathrm{~mm}$ compared to the previous iteration where $\delta=13.6 \mathrm{~mm}$. This suggests that moving the location where the bodyside compression begins by a moderate amount has little influence on the development of the bodyside boundary layer when not combined with a modified body-sidewall corner line, as had been done for Case 2.

\section{Changes from Case 6 to Case 7}

A bump was introduced on the bodyside surface as the geometry change between Case 6 and Case 7 . The reasoning behind the change was to push the boundary layer build-up away from the center and towards the sides. Although an attempt was made to make only single geometry changes per iteration, it was necessary in this case to adjust two of the controlling ribs in order to make the change. The cowl leading edge rib and the notch rib were altered.

The metrics for the Case 7 inlet are recorded in Table 2. Figure 17 shows the flow field for this case. Most of the values for the inlet metrics are comparable to previous iterations. However, the boundary layer thickness, $\delta$, has again increased from the previous iteration to a value of $18.3 \mathrm{~mm}$. This is a larger value than the baseline case. In this geometry, the bump begins at the forward turn on the bodyside wall but is blended 


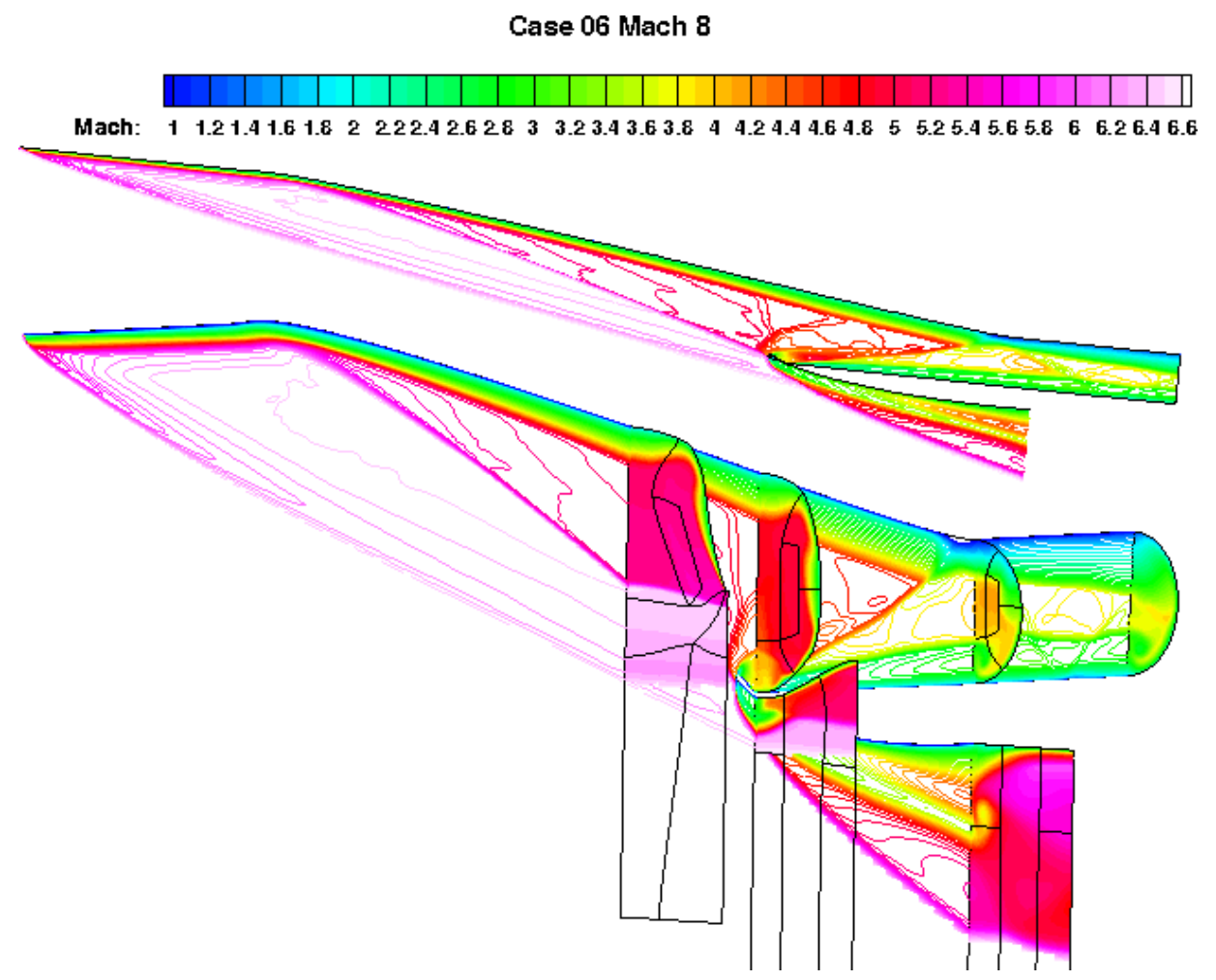

Figure 16. Contours of Mach number for Case 6 with $M_{0}=8$. Flow is from left to right.

away into the throat ellipse shape. The bump has disappeared from the bodyside surface by the time the throat is reached. It seems that initially the boundary layer growth is delayed by the bump. However, when the bump is later blended away, the boundary layer buildup migrates back towards the centerline, and in such a way as to actually increase the thickness along the centerline at the throat. Along with the increase of the boundary layer thickness, the boundary layer area ratio has also increased significantly. This increase suggests that rather than just a migration of boundary layer from some other location on the perimeter to the centerline, there has been an overall increase in boundary layer thickness.

\section{Changes from Case 7 to Case 8}

In Case 8, the orientation of the ellipse switched directions, as had been done in the exploratory design stage. In this case, the aspect ratio was changed from 0.681 to 1.75 . The rib at the corner of the cowl leading edge was also adjusted to accommodate the change in ellipse orientation. However, the lower half of the notch rib was not altered as in Case 4, which was done to produce less compression at the notch and a flatter internal cowl.

Table 2 contains the post-processed results from the flow field analysis for Case 8. In this case, there has been a significant degradation in the total pressure recovery. The boundary layer thickness on the body centerline has been reduced significantly in this iteration: $\delta=12.6 \mathrm{~mm}$. This is in contrast to the previous iteration (Case 7) where $\delta=18.3 \mathrm{~mm}$. In both cases, the inlet had a bump. Therefore, the reduction in boundary layer buildup is largely due to the change in orientation of the ellipse. This stands to reason as this configuration has more aggressive compression on the bodyside surface, and the sidewalls give comparatively less compression. This relieves the build-up of boundary layer on the inlet centerline. The contours of Mach number for this case are shown in Figure 18.

\section{Discussion}

In summarizing the results from all of the on-design cases, there are three main points that can be made. Firstly, it is possible to positively influence a certain inlet metric - in this case, boundary layer thickness on the bodyside centerline - by changes to the parametrized inlet geometry as part of the design toolset. 


\section{Case 07 Mach 8}

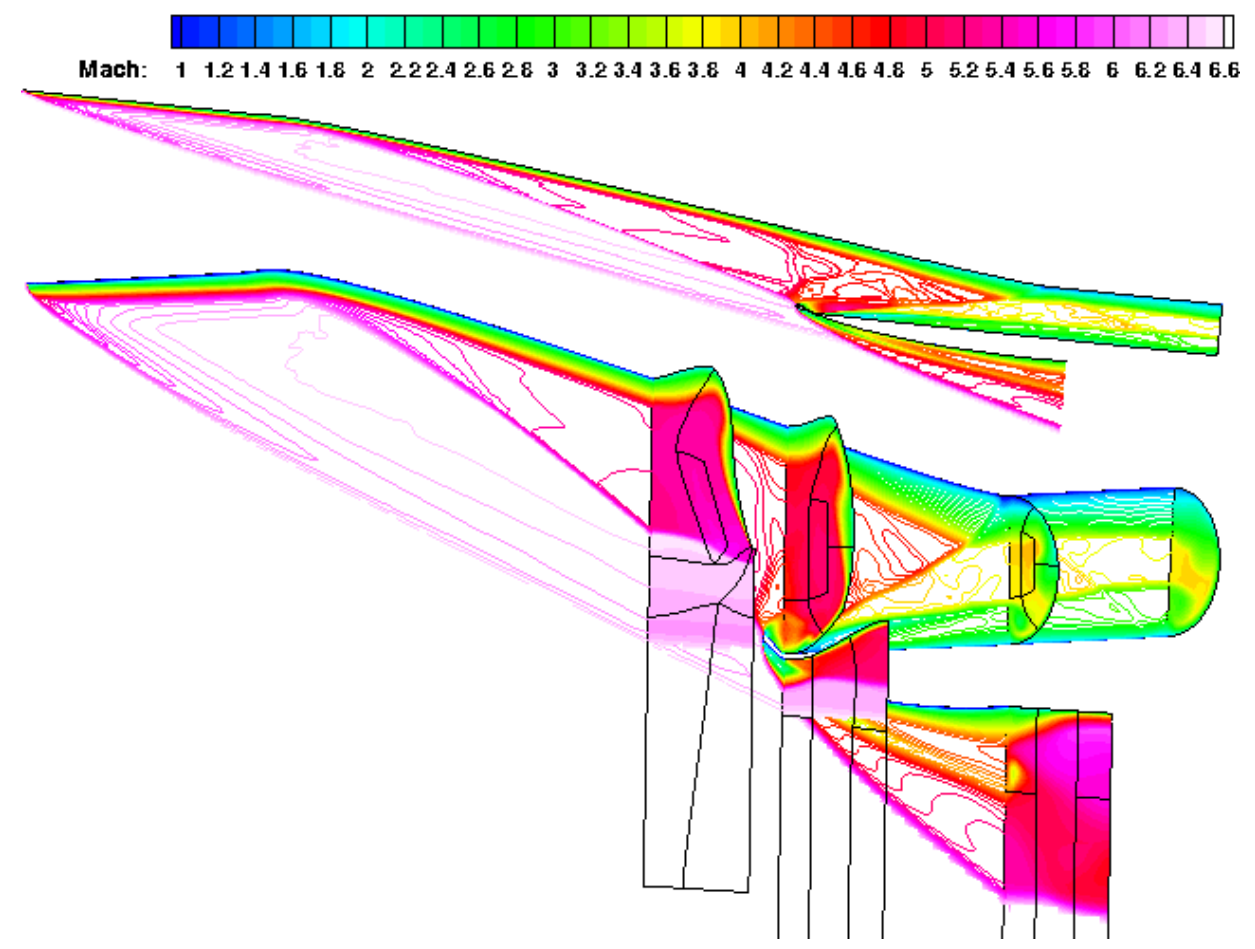

Figure 17. Contours of Mach number for Case 7 with $M_{0}=8$. Flow is from left to right.

In the best case, the boundary layer thickness was reduced from from $15.0 \mathrm{~mm}$ in Case 1 to $11.8 \mathrm{~mm}$ in Case 4; a reduction of about $20 \%$. When re-performing the design iterations in a step-by-step manner, the use of an ellipse with its major axis aligned in the spanwise direction (aspect ratio greater than 1.0) had the largest influence on reducing the boundary layer thickness. This shape change allowed greater bodyside compression while simultaneously reducing the amount of sidewall compression.

Secondly, the integrated performance metrics remained largely unchanged across all iterations, that is, the values were not influenced significantly by local geometry changes. In particular, the boundary layer area ratio, $A_{\delta} / A_{c}$, only varied by about $15 \%$ between its smallest and largest values. This suggests that although the boundary layer thickness on the body centerline was reduced in some cases, the portion of low momentum boundary layer flow was pushed to other parts of the surface. The fact that the performance metrics were relatively constant may be viewed in a positive light. It shows that if one has a good baseline design, then it is possible to make considerable changes to the geometry to accommodate vehicle design constraints without adversely affecting the inlet performance. On the other hand, this result suggests that a poor baseline design would not be a good candidate with which to begin a formal optimization process because local geometry changes do not seem to have large effects on global measures of performance.

Thirdly, this study has demonstrated the hazards of attempting to improve a single local viscous performance metric. To date, no geometry modification of the baseline design has simultaneously improved all performance metrics. In particular, another local viscous performance metric, $\tau_{\min }$, was the best (highest) for the baseline design. Rather than attempt to use correlations for isolator back-pressure capability, it is recommended that the effects of high combustor pressures be included in the computational modelling during the inlet design. The eventual optimization function should then include several performance metrics.

The trends in the perfomance metrics between iterations for the on-design cases are generally observed to be the same for the off-design cases. For example, when there was a reduction of boundary layer thickness at the on-design Mach number, there was a corresponding reduction of boundary layer thickness for the off-design cases. This is pleasing as it means that improvements made at the on-design Mach number did not adversely affect the off-design performance. 


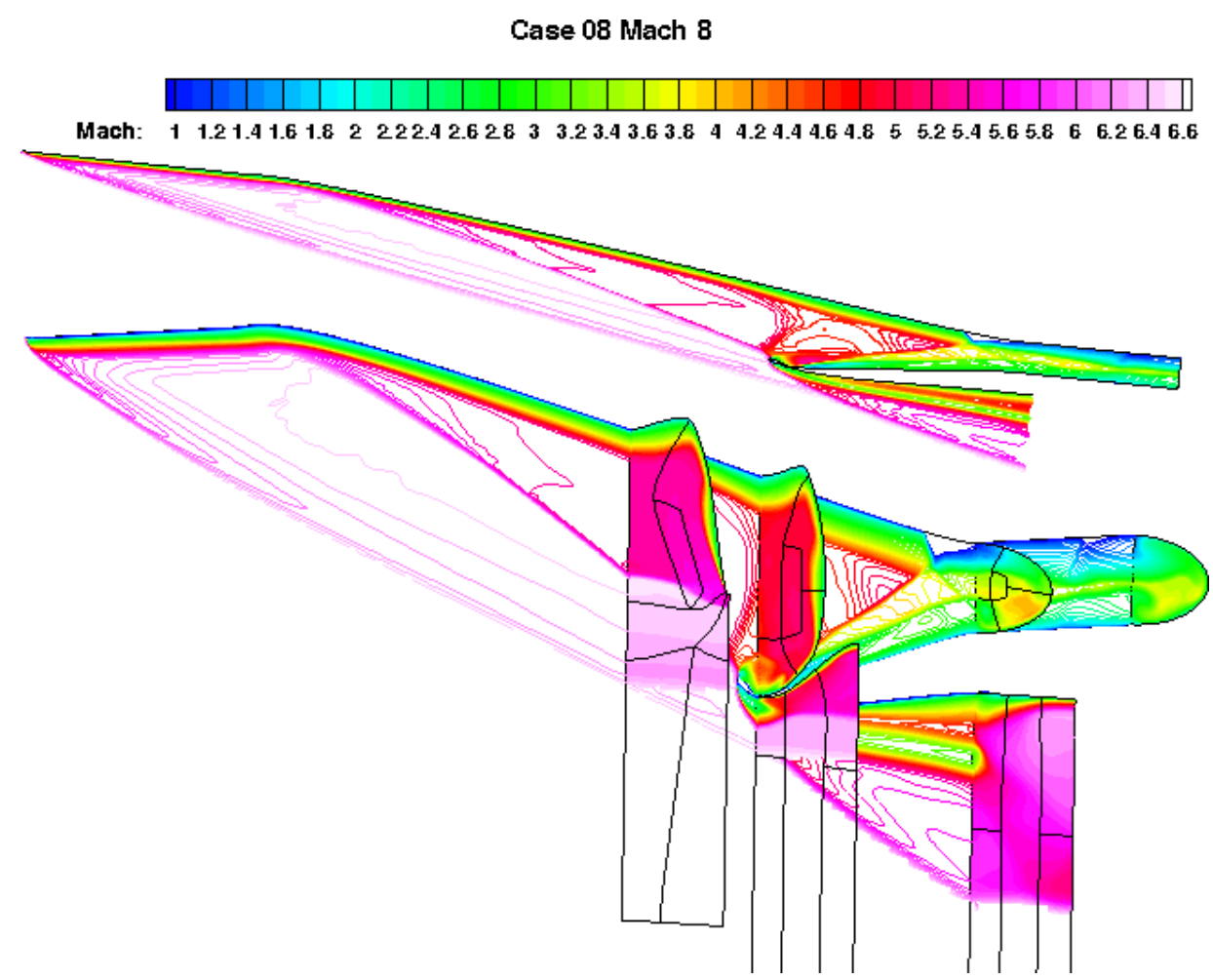

Figure 18. Contours of Mach number for Case 8 with $M_{0}=8$. Flow is from left to right.

\section{Conclusion}

This paper reported on the development of a toolset to design and rapidly analyze REST-class hypersonic inlets. The REST inlets are represented by a parametrized geometry. The inlet designer makes changes to the parameters and is able to instantly view the resulting inlet geometry changes. The inlet geometry is output as a surface and then a structured volume grid is built for flow field analysis. The final step is to analyze the inlet flow with a Navier-Stokes CFD calculation. Presently, the rate-limiting step that involves human intervention is the need to alter the wireframe topology for generating a new structured grid between design iterations.

In the second part of the paper, the toolset was applied to the design of a REST inlet of about $1 \mathrm{~m}$ length for a $M_{0}=8$ cruise application. The challenge was to use the design tools to manually improve a certain inlet metric by morphing the inlet geometry and learning about the metric responses. In this case, the focus was on reducing the thickness of the boundary layer on the bodyside centerline. The design exercise was attempted in two stages: (1) an exploratory stage, and (2) a step-by-step stage. In the exploratory stage, the designers made various intuitive changes to the inlet geometry and assessed the effects of those changes in terms of the flow field properties. A successful outcome of this stage was that the boundary layer thickness was reduced by about $20 \%$ of its original value and significantly reduced total drag. In the second stage, the lessons learned about geometry changes in the first stage were used to step through the single geometry changes, iteration by iteration. By using this approach, the hope was to identify those geometry changes with the largest influence on reducing the boundary layer buildup on the bodyside surface. It was found that changing the orientation of the ellipse so as to give greater bodyside compression and less sidewall compression had the largest influence on reducing the bodyside boundary layer thickness.

As a final note, the moderate geometry changes made for design iterations 3-7 did not produce large changes in the integrated metrics of the inlet. In other words, the morphing of the inlet produced local changes in features but did not favorably or adversely affect the integrated inlet performance. This may be viewed in a positive light: it is possible to make local changes to a REST inlet geometry to suit design constraints without significantly changing its global performance metrics. 


\section{Acknowledgment}

The authors would like to thank Hilmi Alkamhawi of TechnoSoft, Inc. for programming the AML module which handles the parametrized geometry form of the REST inlets.

\section{References}

${ }^{1}$ Smart, M. K., "Design of three-dimensional hypersonic inlets with rectangular-to-elliptical shape transition," Journal of Propulsion and Power, Vol. 15, No. 3, 1999, pp. 408-416.

${ }^{2}$ Ferlemann, P. G. and Gollan, R. J., "Parametric Geometry, Structured Grid Generation, and Initial Design Study for REST-class Hypersonic Inlets," JANNAF Airbreathing Propulsion Subcommittee Meeting, La Jolla, California, 2009.

${ }^{3}$ AML (Adaptive Modelling Language) from Technosoft Inc., Cincinnati, OH, http://www.technosoft.com.

${ }^{4}$ VULCAN, http://vulcan-cfd.larc.nasa.gov.

${ }^{5}$ White, J. A. and Morrison, J. H., "A Pseudo-temporal Multi-grid Relaxation Scheme for Solving the Parabolized Navier-Stokes Equations," AIAA Paper 99-3360, 1999.

${ }^{6}$ GridPro, Program Development Corporation, White Plains, NY, http://www.gridpro.com.

${ }^{7}$ Gridgen, Pointwise, Inc., Fort Worth, TX, http://www.pointwise.com/gridgen.

${ }^{8}$ Wilcox, D. C., Turbulence Modelling for CFD, DCW Industries, La Cañada, California, 2nd ed., 1998.

${ }^{9}$ Edwards, J. R., "A low-diffusion flux-splitting scheme for Navier-Stokes calculations," Computers and Fluids, Vol. 26, No. 6, 1997, pp. 635-659.

${ }^{10}$ Venkatakrishnan, V., "On the Accuracy of Limiters and Convergence to Steady State Solutions," AIAA Paper 930880, 1993.

${ }^{11}$ Mölder, S. and Romeskie, J. M., "Modular Hypersonic Inlets with Conical Flow," AGARD CP-30, McGill University, 1968.

${ }^{12}$ Baurle, R. A. and Gaffney, R. L., "Extraction of One-Dimensional Flow Properties from Multidimensional Data Sets," Journal of Propulsion and Power, Vol. 24, No. 4, 2008, pp. 704-714. 